\title{
Non-monotonic thermal Casimir force from geometry-temperature interplay
}

\author{
Alexej Weber ${ }^{1}$ and Holger Gies ${ }^{2}$ \\ ${ }^{1}$ Institut für Theoretische Physik, Universität Heidelberg, Philosophenweg 16, D-69120 Heidelberg, Germany \\ ${ }^{2}$ Theoretisch-Physikalisches Institut, Friedrich-Schiller-Universität Jena, Max-Wien-Platz 1, D-07743 Jena, Germany
}

\begin{abstract}
The geometry dependence of Casimir forces is significantly more pronounced in the presence of thermal fluctuations due to a generic geometry-temperature interplay. We show that the thermal force for standard sphere-plate or cylinder-plate geometries develops a non-monotonic behavior already in the simple case of a fluctuating Dirichlet scalar. In particular, the attractive thermal force can increase for increasing distances below a critical temperature. This anomalous behavior is triggered by a reweighting of relevant fluctuations on the scale of the thermal wavelength. The essence of the phenomenon becomes transparent within the worldline picture of the Casimir effect.
\end{abstract}

PACS numbers:

Fluctuations of the radiation field between mesoscopic or macroscopic test bodies give rise to the fascinating Casimir effect [1] - a dispersive quantum and relativistic force phenomenon in the absence of net charges, see 2] for reviews. Experimental verifications [3] typically involve spheres or cylinders and plates. For standard materials, the Casimir force is generally attractive [4] and decreases monotonically with distance. The latter seems intuitively clear from spectral properties of the fluctuations: in this picture, the Casimir effect arises from the difference between the fluctuation spectrum in the presence of the surfaces and that of the trivial vacuum (at infinite surface separation). For increasing separation, the spectrum is expected to monotonically approach the vacuum spectrum, implying a monotonic force depletion.

A first non-monotonic behavior has been observed in a more involved piston-like geometry of two squares moving between metal walls [5]; similar observations hold for two cylinders near a sidewall [6]. Here, the non-monotonic behavior arises from a competition between the TE and TM modes of the electromagnetic fluctuations. Its strength is governed by the dependence of the force on a lateral geometry parameter. The example demonstrates that an unexpected behavior of the Casimir force may occur in the presence of competing scales (in this case: normal and lateral distances).

In this work, we show that a non-monotonic behavior already exists for a single fluctuating scalar obeying Dirichlet boundary conditions on the surfaces (similar to a TM mode in a cavity-like configuration). This anomalous phenomenon requires a nonzero temperature and occurs for the thermal contribution to the Casimir force which we abbreviate by "thermal force" in the following.

This phenomenon is a prime example of the general geometry-temperature or geothermal interplay 7]. As first conjectured in [8], the thermal correction to the Casimir effect can vary qualitatively for different geometries, as both the zero- and finite-temperature Casimir effect are based on the underlying fluctuation spectrum. Analytical and numerical evidence for this geothermal interplay has been collected using perpendicular- or inclined-plates configurations $[7,9,10]$. At low temperatures, the temperature dependence is more pronounced in open geometries which do not exhibit a gap in the relevant part of the fluctuation spectrum. At any small value of the temperature, thermal modes can be excited, inducing a characteristic thermal force. By contrast, geometries with a gap in the spectrum show a suppressed thermal force, as thermal modes can hardly be excited for temperatures below the gap scale.

Typical configurations used in experiments involve spheres or cylinders above a plate which are open geometries without a spectral gap. Investigating the geothermal interplay for these geometries therefore is an urgent problem. For the fluctuating electromagnetic field, first results for the thermal Casimir force in the sphere-plate configuration have recently been obtained [11, 12] using scattering techniques. In the limit of temperature being smaller than both the inverse sphere radius, $T \ll 1 / R$, and the inverse sphere-plate distance, $T \ll 1 / a$, the thermal force in 12] is always attractive for any value of $a / R$ and monotonically decreasing with increasing separation $a$. By contrast, the thermal force derived in [11] using a truncated multipole summation shows a repulsive behavior for smaller distances and becomes attractive at larger distances. From their data, a non-monotonic behavior of the thermal force at larger distances $a / R \gg 1$ and low temperatures $T R \lesssim 1$ can be anticipated. Whereas both studies nicely agree in the limit of low temperature and small spheres, the seeming disagreement beyond the strictly asymptotic validity regimes of the two different expansions requires clarification.

In this work, we demonstrate that non-monotonic thermal forces indeed occur unambiguously for the sphere-plate and cylinder-plate configuration in the lowtemperature region. For simplicity and clarity, we use Dirichlet scalar fluctuations in order to avoid additional complications from competing polarization modes. Most importantly, the occurrence of this anomalous behavior can be understood as a temperature-induced reweighting of different relevant fluctuations in a given geometry. This is at the heart of the geothermal interplay. 


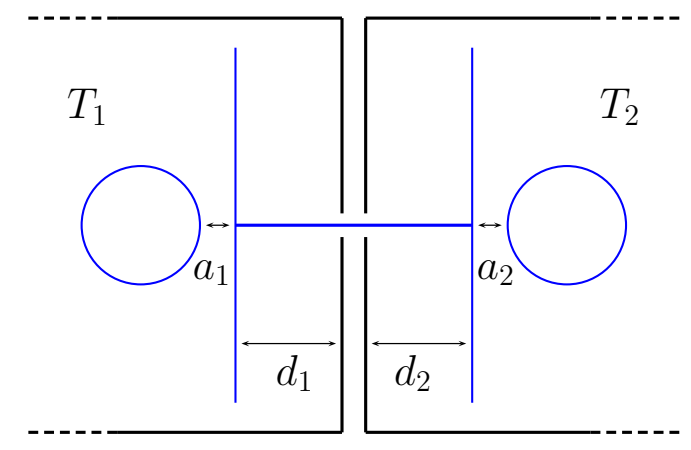

FIG. 1: Setup for a measurement of the thermal force for the sphere-plane configuration: by tuning the sphere positions to identical distances $a_{1}=a_{2}$, the zero-temperature force on the tightly connected pair of plates cancels. Placing the two sphere-plate subsystems into two heat baths at different temperatures $T_{1}$ and $T_{2}$, the different thermal forces induce a net force on the pair of plates. We assume that all distances between the (blue) Casimir surfaces and the (black) heat-bath boundaries are large, e.g., $d_{1,2} \gg a_{1,2}$, such that corresponding Casimir interactions can be neglected.

The origin of this phenomenon can most intuitively be understood in the worldline picture of the Casimir effect [13]. The worldline formalism maps the standard spectral Casimir problem to a Feynman path integral over closed worldlines in position space. These worldlines can be viewed as the space trajectories of quantum fluctuations within a given Casimir geometry. In addition to this intuitive picture, the worldline approach offers a powerful tool for numerical as well as analytical studies. E.g., for the sphere/cylinder-plate configuration at zero temperature, a coherent understanding of the Casimir force has emerged from worldline studies [14] on the one hand and scattering techniques on the other hand 15 18]. A similar consensus has been reached for "edge effects" [9, 19]. Also for more involved geometries, the worldline picture directly provides for a qualitative understanding [20].

The Casimir free energy induced by a fluctuating Dirichlet scalar reads for any configuration [13, 14]

$$
E_{\mathrm{c}}=-\frac{1}{32 \pi^{2}} \int_{0}^{\infty} \frac{\mathrm{d} \mathcal{T}}{\mathcal{T}^{3}} \sum_{n=-\infty}^{\infty} e^{-\frac{n^{2} \beta^{2}}{4 \mathcal{T}}} \int_{\mathbf{x}_{\mathrm{CM}}}\langle\Theta[\boldsymbol{\gamma} ; \mathcal{T}]\rangle
$$

Here, $\langle\ldots\rangle$ denotes an average over an ensemble of worldlines $\gamma$ with Gaußian velocity distribution with a common center of mass $\mathbf{x}_{\mathrm{CM}}$. The auxiliary propertime parameter $\mathcal{T}$ acts as a spatial scaling factor and governs the size of the worldlines. The sum over winding number $n$ counting the round trips of a worldline around the finite-temperature torus takes care of thermal fluctuations, with the $n=0$ term corresponding to the zerotemperature $T=1 / \beta \rightarrow 0$ result. The $\Theta$ functional obeys $\Theta=1$ if a given worldline intersects two or more interacting surfaces, and $\Theta=0$ otherwise.

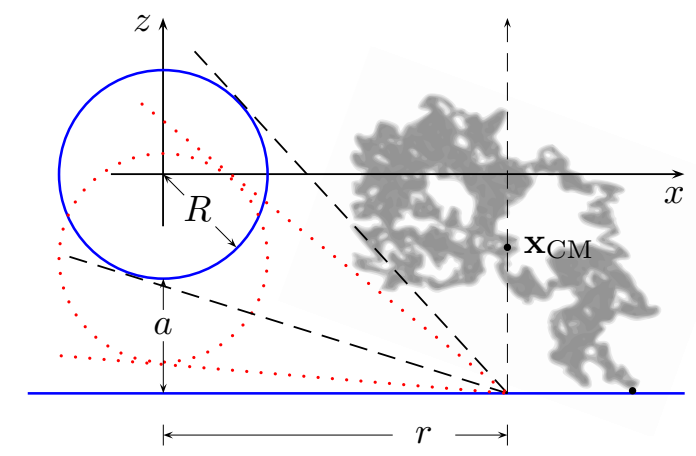

FIG. 2: Sketch of the sphere-plate configuration. During the propertime integration, the worldline is always attached to the plate, while all its points move on rays originating from the projection of its center of mass $\mathbf{x}_{\mathrm{CM}}$ on the plate at polar coordinate $r$. Only points lying inside a cone wrapping around the sphere with the tip of the cone at $r$ (thick dashed lines) pass through the sphere for increasing $\mathcal{T}$ and thus contribute to the Casimir force. In general, for every $r \gg R$ there exists a maximal separation $a$, such that the sphere can be intersected by the worldline. If the sphere separation is reduced (dotted red circle), it can become invisible for a given worldline, such that the worldline stops contributing to the Casimir force. This induces the phenomenon that the thermal force can increase for increasing separation.

In the following, we are exclusively interested in the thermal contribution $n \neq 0$, serving as the interaction potential for the thermal force, $F_{T}=-d E_{\mathrm{c}} /\left.d a\right|_{n \neq 0}$; here, $a$ is a distance parameter between disjoint bodies. For instance, for a sphere above a plate, a configuration that allows to cancel the zero-temperature forces is sketched in Fig. 1. Alternatively, the zero-temperature force could be balanced by applying suitable electrostatic potentials to a single-sphere setup. We stress that the thermal-force phenomena discussed in the following are dominated by the corresponding zero-temperature forces in the standard setups where $a \ll R$. After removing the zero- $T$ contribution, the thermal force reads

$$
F_{T}=-\frac{1}{8 \pi} \sum_{n=1}^{\infty} \int_{0}^{\infty} \mathrm{d} \mathcal{T} \frac{e^{-\frac{n^{2} \beta^{2}}{4 \mathcal{T}}}}{\mathcal{T}^{3}} \int_{0}^{\infty} \mathrm{d} r r\left\langle\Theta_{\mathrm{S}}\right\rangle,
$$

where $r$ is the polar center-of-mass coordinate on the plate. $\Theta_{\mathrm{S}}$ measures whether a worldline which is attached to the plate also intersects with the sphere for certain scaling sizes $\sim \mathcal{T}$, see Fig. 2 For the cylinder-plate case, the factor of $r$ has to be replaced by the (infinite) length $L_{y}$ of the cylinder divided by $\pi$.

The geothermal effect can easily be understood in the worldline picture: at zero temperature, the Casimir force is generically dominated by small propertimes, i.e., small worldlines with a minimal extent such that the worldlines can intersect with both surfaces. As a consequence, the energy density is typically peaked in the region near 


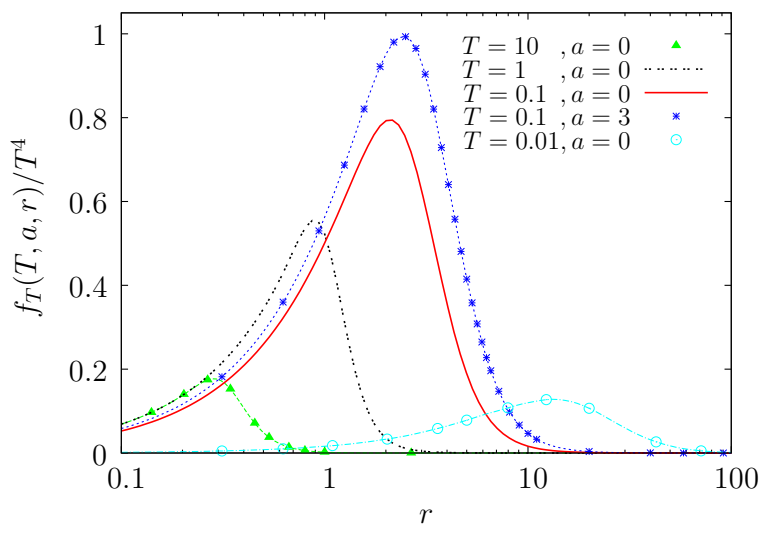

FIG. 3: Radial thermal force density $f_{T}(T, a, r)$ for a sphere above a plate for different temperatures $T$ in units of $R=1$. The peak position as a function of $r$ increases with decreasing temperature. The statistical error is below one percent.

minimal separation. By contrast, the peak of the finitetemperature propertime factor $\sim e^{-n^{2} \beta^{2} /(4 \mathcal{T})} / \mathcal{T}^{3}$ moves to larger $\mathcal{T}$ values for decreasing temperature. Therefore, as larger worldlines can contribute, the free-energy density is potentially distributed over a larger region of space. Whether or not this broadening occurs depends on the details of the geometry, as worldlines at larger distances still have to intersect the Casimir surfaces.

In Fig. 3. we plot the radial distribution of the thermal force density for various temperature values. Its peak position increases with decreasing temperature. This corresponds to the fact that low temperatures can still excite long-wavelength modes if the spectrum is not gapped. Figure 3 also demonstrates that any local approximation of the Casimir force such as the proximity force approximation (PFA) is generically bound to fail for a proper description of the geometry-temperature interplay; see, however, 21] for semitransparent surfaces. For experiments at low temperature, our results indicate that the idealized sphere-plate configuration requires the plate to be much larger than the sphere. Otherwise thermal edge effects have to be accounted for, being more severe than edge effects at $T=0$.

In addition to the potential delocalization of the thermal force density, a second geometric mechanism is operative for the non-monotonic behavior of the thermal Casimir force: in the worldline picture formalized by Eq. (2), the Casimir force arises from all worldlines that are attached to the plate and intersect the sphere. Consider a worldline with center of mass polar coordinate $r$ in the sphere-plate geometry at separation $a$, see Fig. 2] Upon integrating over propertime $\mathcal{T}$, the size of the worldlines are scaled but the center of mass projection onto the sphere stays fixed at $r$. Therefore, the worldline points that eventually intersect the sphere have to lie inside a cone with its tip attached to the plate at $r$, wrapping around the sphere, see Fig. 2 .

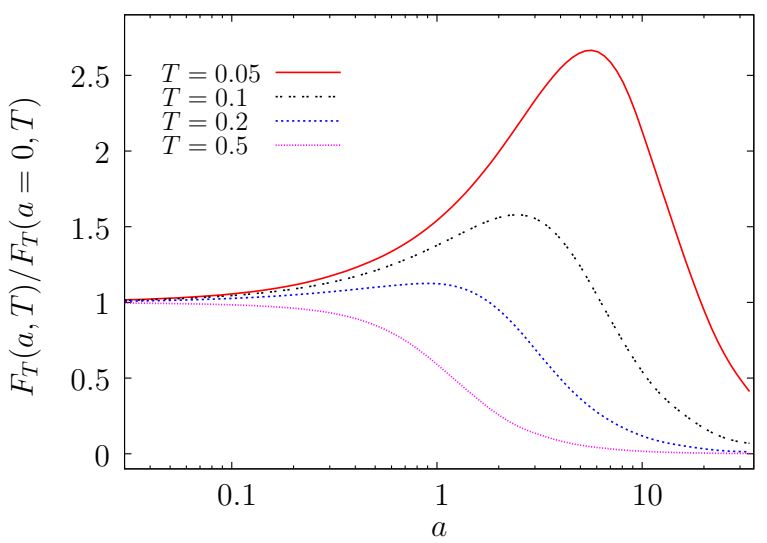

FIG. 4: Thermal Casimir force of a sphere for various temperatures $T$ and $R=1$, normalized to the thermal force at $a=0$. For sufficiently small temperatures, the absolute value of the thermal force $F_{T}$ first increases with increasing $a$. For $T \lesssim 0.05$ the small- $a$ behavior of the normalized curves are well described by $1+a\left(2.68 R T^{4}-R^{2} 15.7 T^{5}\right) / F_{T}(a=0, T)$. The statistical error is on the order of the curve thickness.

Let us now reduce the sphere-plate separation $a$ for a given worldline. As this moves the corresponding cone towards the plate (red dotted lines in Fig. 2), all points of a given worldline may drop out of the cone such that this worldline no longer contributes to the Casimir force. This is the mechanism that potentially reduces the Casimir force for smaller separations.

While this geometric argument is independent of any temperature, this loss mechanism of relevant fluctuations is negligible at zero temperature: the effect is outweighed by small worldlines intersecting sphere and plate near the point of closest separation which dominate the zero-temperature Casimir force. By contrast, the finitetemperature propertime factor $\sim e^{-n^{2} \beta^{2} /(4 \mathcal{T})} / \mathcal{T}^{3}$ favors the contribution of larger worldlines at low temperature and thus emphasizes the relevance of the loss mechanism at larger separations. Whether or not a non-monotonic thermal force law arises then is a competition between small worldlines in the region of close separation and large worldlines on the scale of the thermal wavelength. If the contribution of the latter to the thermal force is dominant, the thermal force can increase for increasing distance as more and more worldlines can contribute, i.e., become relevant fluctuations.

Worldline numerical results for the thermal force for a sphere above a plate as a function of separation $a$ are displayed in Fig. 4. We have used up to 40000 worldlines discretized by $2 \cdot 10^{6}$ points each. As the limit of zero separation $a \rightarrow 0$ exists for thermal forces according to a general argument [10], we normalize the thermal force relative to the $a \rightarrow 0$ limit. All dimensionful scales are normalized to the sphere radius which we set to $R=1$ in the following. For temperatures larger than a critical temperature $T>T_{\text {cr }} \simeq 0.34(1) / R$, we observe an attrac- 
tive thermal force which decreases monotonically for increasing sphere-plate separation $a$ and thus satisfies standard expectations. For smaller temperatures $T<T_{\text {cr }}$, the thermal force first increases for increasing separation, develops a maximum and then decreases to zero for infinite separation. The peak position increases with increasing thermal wavelength, i.e., inverse temperature. In all cases, the force remains attractive. As an example, room temperature $T=300 \mathrm{~K}$ is critical for spheres of radius $R \simeq 2.6 \mu \mathrm{m}$, supercritical for larger and subcritical for smaller spheres. At $T=70 \mathrm{~K}$ and $R=1.6 \mu \mathrm{m}$, the thermal force increases up to $a \simeq 9 \mu \mathrm{m}$.

For small $T \ll 1 / R$ and $a \ll R$, our numerical data is compatible with an expansion of the type

$$
F_{T}=c_{2} R^{2} T^{4}+c_{3} a R T^{4}+\mathcal{O}\left((a / R)^{2},(T R)^{5}\right),
$$

where $c_{2} \approx-3.96(5)$ and $c_{3} \approx-2.7(2)$. The absence of terms $\sim c_{0} R T^{3}$ or $c_{1} a T^{3}$ is in agreement with scaling arguments 22] and numerically confirmed in the regime $T>0.01$ where data is available.

Since $c_{2}$ and $c_{3}$ have the same sign, the absolute value of the thermal Casimir force $F_{T}$ increases with increasing $a$ for sufficiently small $a$ and $T$. In passing, we note that the high-temperature limit $T \gg 1 / R$ agrees with the PFA prediction for $a \rightarrow 0: F_{T}=-\zeta(3) R T^{3} / 2$. At low temperatures, the PFA disagrees with the full result even in the $a \rightarrow 0$ limit, as observed in 10 .

The case of a cylinder above a plate is different from the sphere-plate case in two respects: first, the missing polar measure factor $r$ reduces the weight of distant worldlines. Second, the probability for a given worldline to intersect the infinitely long cylinder is larger than for a sphere. We observe that these two effects balance each other, leading again to a $T^{4}$ behavior at low $T$ :

$$
F_{T} / L_{y}=c_{2} R T^{4}+c_{3} a T^{4}+\ldots,
$$

where $c_{2} \approx-1.007(7)$ and $c_{3} \approx-0.41(4)$. The coefficients again have the same sign, implying that the thermal force increases with $a$ for sufficiently small $a$ and $T$. The behavior is then similar to Fig. 4. For the critical temperature, we obtain $T_{\mathrm{cr}} \approx 0.31(1) / R$. At high temperatures and $a=0$, the thermal force agrees with the PFA prediction as expected, $F_{T} \approx-0.278 \sqrt{R} T^{\frac{7}{2}}$.

To summarize, we have shown that the thermal contribution to the Casimir force for the standard sphere-plate and cylinder-plate geometry develops a non-monotonic behavior already in the simple case of a fluctuating Dirichlet scalar. For temperatures below a critical value and for small distances, the thermal force increases for increasing separation. This phenomenon is a prime example for the geometry-temperature interplay in open geometries. It has a simple geometric interpretation in the worldline picture and can reliably be computed in the numerical worldline approach.

We stress that the case of electromagnetic fluctuations can develop yet another thermal-force behavior due to possibly competing polarization modes. For a quantitative comparison with experimental data, a nontrivial interplay between geometry, temperature as well as dielectric material properties can be expected; pioneering first results on this interplay have been obtained in [11].

We thank T. Emig for suggesting the setup in Fig. 1 and E. Elizalde for useful discussions. We have benefited from activities within ESF Research network CASIMIR. We acknowledge support by the Landesgraduiertenförderung Baden-Württemberg, by the Heidelberg Graduate School of Fundamental Physics, and by the DFG under Gi 328/3-2 and Gi 328/5-1.

[1] H.B.G. Casimir, Kon. Ned. Akad. Wetensch. Proc. 51, 793 (1948).

[2] K. A. Milton, "The Casimir effect" River Edge, USA: World Scientific (2001); M. Bordag, G. L. Klimchitskaya, U. Mohideen and V. M. Mostepanenko, Int. Ser. Monogr. Phys. 145, 1 (2009).

[3] S. K. Lamoreaux, Phys. Rev. Lett. 78, 5 (1997); U. Mohideen and A. Roy, Phys. Rev. Lett. 81, 4549 (1998); H.B. Chan et al., Science 291, 1941 (2001); G. Bressi et al., Phys. Rev. Lett. 88, 041804 (2002); R.S. Decca et al., Phys. Rev. Lett. 94, 240401 (2005).

[4] O. Kenneth and I. Klich, Phys. Rev. Lett. 97, 160401 (2006).

[5] A. Rodriguez et al., Phys. Rev. Lett. 99, 080401 (2007); Phys. Rev. A 76, 032106 (2007).

[6] S.J. Rahi, T. Emig, R.L. Jaffe, and M. Kardar, Phys. Rev. A 78, 012104 (2008).

[7] H. Gies and K. Klingmuller, J. Phys. A 41, 164042 (2008).

[8] A. Scardicchio and R. L. Jaffe, Nucl. Phys. B 743 (2006) 249.

[9] A. Weber and H. Gies, Phys. Rev. D 80, 065033 (2009).

[10] H. Gies and A. Weber, arXiv:0912.0125 [hep-th].

[11] A. Canaguier-Durand, P.A. Maia Neto, A. Lambrecht, and S. Reynaud, arXiv:0911.0913.

[12] M. Bordag and I. Pirozhenko, arXiv:0912.4047.

[13] H. Gies, K. Langfeld and L. Moyaerts, JHEP 0306, 018 (2003); arXiv:hep-th/0311168.

[14] H. Gies and K. Klingmuller, Phys. Rev. Lett. 96, 220401 (2006); Phys. Rev. D 74, 045002 (2006).

[15] A. Bulgac, P. Magierski and A. Wirzba, Phys. Rev. D 73, 025007 (2006).

[16] T. Emig, R. L. Jaffe, M. Kardar and A. Scardicchio, Phys. Rev. Lett. 96 (2006) 080403.

[17] M. Bordag, Phys. Rev. D 73, 125018 (2006); Phys. Rev. D 75, 065003 (2007).

[18] K. A. Milton and J. Wagner, Phys. Rev. D 77, 045005 (2008); J. Phys. A 41, 155402 (2008).

[19] H. Gies and K. Klingmuller, Phys. Rev. Lett. 97, 220405 (2006); N. Graham, A. Shpunt, T. Emig, S. J. Rahi, R. L. Jaffe and M. Kardar, arXiv:0910.4649 [quant-ph]; D. Kabat, D. Karabali and V. P. Nair, arXiv:1002.3575.

[20] M. Schaden, Phys. Rev. Lett. 102, 060402 (2009).

[21] K. A. Milton, P. Parashar, J. Wagner and K. V. Shajesh, arXiv:0909.0977 [hep-th].

[22] A. Weber and H. Gies, in preparation (2010). 